Fetal Diagnosis and Therapy
Fetal Diagn Ther 2014;36:196-201

DOI: $10.1159 / 000360854$
Received: December 17, 2013

Accepted after revision: February 20, 2014

Published online: August 15, 2014

\title{
Circulating mRNA for the PLAC1 Gene as a Second Trimester Marker (14-18 Weeks' Gestation) in the Screening for Late Preeclampsia
}

\author{
Margherita Zanello ${ }^{a}$ Akihiko Sekizawab Yuditiya Purwosunu ${ }^{c}$ \\ Alessandra Curti ${ }^{\mathrm{a}}$ Antonio Farina $^{\mathrm{a}}$ \\ a Division of Prenatal Medicine, Department of Medicine and Surgery DIMEC, University of Bologna, Bologna, Italy; \\ ${ }^{b}$ Department of Obstetrics and Gynecology, Showa University School of Medicine, Tokyo, Japan; ' Department of \\ Obstetrics and Gynecology, University of Indonesia, Cipto Mangunkusumo National Hospital, Jakarta, Indonesia
}

\section{Key Words}

Molecular screening for preeclampsia $\cdot$ mRNA for the

PLAC1 gene $\cdot$ Patient-specific risk - Multivariable screening at the same level of FPR. PLAC1 alone yielded a DR of $30.2 \%$. Conclusion: In late $\mathrm{PE}$, molecular markers can be used to improve the DR of screening and can be a valid option for the biochemical approach.

(c) 2014 S. Karger AG, Basel

\begin{abstract}
Objective: To develop a model for prediction of late preeclampsia (PE; which develops at or after 34 weeks' gestation) based on maternal history and characteristics, mean arterial pressure (MAP), and circulating levels of mRNA for the placenta-specific 1 (PLAC1) gene in maternal plasma at 14-18 weeks' gestation. Method: This was a screening study of singleton pregnancies at 14-18 weeks' gestation including 43 women that subsequently developed PE and 200 that were unaffected by PE. A Gaussian model was fitted to the log distribution of the multiple of the median (log MoM) PLAC1 mRNA in the PE group and in the unaffected group. Likelihood ratios for log MoM of circulating levels of mRNA for the PLAC1 gene were used to combine the a priori risk from maternal characteristics with MAP to produce patient-specific risks for each case. Results: Screening by maternal characteristics (including BMI, woman's mother's history of PE, previous $\mathrm{PE}$, and parity) (a priori risk) and MAP detected $46.8 \%$ of all cases of late PE at a fixed false-positive rate (FPR) of $10 \%$. The addition of PLAC1 yielded a detection rate (DR) of $62.8 \%$
\end{abstract}

\section{Introduction}

Preeclampsia (PE) arises as a complication in $2-8 \%$ of pregnancies and remains one of the main causes of maternal and fetal death and morbidity [1]. In developed countries, PE is the primary cause of maternal admission to intensive care units and causes approximately $15 \%$ of all pregnancy-related deaths [2]. As PE is known to have a long preclinical phase before the onset of clinical symptoms in later gestation, clinical prediction offers the possibility of redirecting maternal and prenatal care in highrisk pregnancies [3]. The discovery of circulating fetal/ placental RNA in maternal plasma has enabled the development of several promising approaches for noninvasive evaluation of placental function [4]. Placenta-specific 1 (PLAC1) is an X-linked gene with expression restricted primarily to cells derived from trophoblast lineage during embryonic development [5]. PLAC1 localizes to a region

\section{KARGER}

E-Mail karger@karger.com

www.karger.com/fdt
(C) 2014 S. Karger AG, Basel

1015-3837/14/0363-0196\$39.50/0
Antonio Farina, $\mathrm{MD}, \mathrm{PhD}$

Department of Medicine and Surgery DIMEC

Division of Prenatal Medicine, University of Bologna

IT-40126 Bologna (Italy)

E-Mail antonio.farina@unibo.it 
of the X chromosome and is essential for normal placental and embryonic development [6]. PLAC1 has previously been evaluated as a potential marker of genetic and gestational disorders, and many papers have reported higher levels of PLAC1 in PE patients than in a healthy population [7-10]. In the past years a consistent number of studies have reported the development of several statistical models for the prediction of PE long before its clinical onset. Most of them involve maternal history, biophysical parameters, Doppler of uterine arteries, and biochemical markers in the prediction of any kind of PE, including early and late onset. For example, in MEDLINE there are more than 100 papers that present results obtained on a huge amount of data. Even if it is a hard challenge to summarize the results of so many articles, the detection rate (DR) for PE ranges from about 45 to 55\% at a false-positive rate (FPR) of $10 \%$ by using maternal history plus mean arterial pressure (MAP), and various combinations of biochemical factors yield a DR around $90 \%$ at the same FPR with a much better degree of prediction for the early onset of PE [11]. On the other hand, our group has already reported risk estimation for PE by means of molecular markers $[12,13]$. Although the number of cases enrolled to build up a predictive model by means of molecular markers was small in comparison with that used in the studies on biochemical markers, the DR of various panels of mRNAs species with and without maternal factors has been quoted as about $80-85 \%$ at $10 \%$ FPR $[12,13]$.

However, until now no papers have attempted to combine the so-called a priori risk derived by some equations with the risk obtained by the Gaussian distribution of mRNA species and expressed as a likelihood ratio (LR). In the medical literature, in fact, only papers that used a combination of biochemical markers and/or Doppler measurements with a panel of equations derived by anamnestic and biophysical maternal factors are present.

In this paper we used a risk model derived from maternal history plus MAP distribution [14] combined with mRNA for PLAC1 at 14-18 gestational weeks, using part of the data already published by Purwosunu et al. [15] in the American Journal of Obstetrics and Gynecology in 2009. In particular, in that paper, 7 mRNAs species, including PLAC1 were dosed in a population of 310 controls and $62 \mathrm{PE}$ cases between 15 and 20 weeks' gestation, including cases of mild PE, severe PE, and HELLP syndromes. The DR at a fixed 5\% FPR was calculated for each mRNA, and PLAC1 yielded a significant discrimination between cases and controls.

Circulating mRNA for PLAC1 in the

Screening for Late Preeclampsia
We decided to dose PLAC1 in the second trimester since the low degree of placental insufficiency typical of late PE would not allow the prediction of the disease. The aim of the present study was to combine, for the first time, maternal factors plus a molecular marker for the risk estimation of late PE by means of the same parametric algorithm used in biochemical screening to allow a possible comparison of the two approaches.

\section{Materials and Methods}

The women were examined between mid-2005 and 2006, and comprised singleton pregnancies that visited the Department of Obstetrics and Gynaecology, University of Indonesia, at Cipto Mangunkusumo National Hospital. Singleton pregnant women without any preexisting medical diseases including chronic hypertension and pregravid diabetes at screening or antenatal complications at the time of blood drawing were invited to participate. The pregnancies were dated by ultrasound, which was performed during the first trimester (11-13 weeks). All women provided informed consent to join in the study that was approved by the Institutional Research Ethics Committee. From the original study [15], we excluded the patients who developed a HELLP syndrome and those whose follow-up was not complete. Again, only the women within the interval 14-18 weeks' gestation were considered. Forty-three cases of PE were therefore retrospectively matched with 200 normal pregnancies in a 1:5 match for gestational age at the time of enrollment. For 15 PE cases, however, only 4 controls were available. Data on pregnancy outcomes were collected from the hospital maternity records. Diagnosis of PE was made according to the criteria of the International Society for the Study of Hypertension in Pregnancy [16]. Hypertension is defined as systolic blood pressure $\geq 140 \mathrm{~mm} \mathrm{Hg}$ and/or diastolic blood pressure $\geq 90 \mathrm{~mm} \mathrm{Hg}$ on at least two occasions $4 \mathrm{~h}$ apart developed after 20 weeks' gestation in previously normotensive women. PE, on the other hand, is characterized by hypertension with proteinuria $\geq 300 \mathrm{mg}$ in $24 \mathrm{~h}$, or two readings of at least ++ on dipstick analysis of midstream or catheter urine specimens if no 24-hour collection is available. We defined late PE as occurring after 34 weeks' gestation (which is also mostly associated with normal or slightly increased uterine resistance index, a low rate of fetal involvement, and more favorable perinatal outcomes).

Processing of blood samples has been described previously [17]. In brief, in the second trimester (14-18 weeks' gestation) 7 -ml peripheral blood samples were collected in EDTA-containing tubes and centrifuged at $1,600 \mathrm{~g}$ for $10 \mathrm{~min}$ at $4{ }^{\circ} \mathrm{C}$ twice within $1 \mathrm{~h}$ from collection. Molecular analysis was performed at the Department of Obstetrics and Gynecology at Showa University School of Medicine, Tokyo, Japan. Total RNA was extracted from $1.6 \mathrm{ml}$ of harvested plasma. The plasma was mixed with $2 \mathrm{ml}$ of Trizol LS reagent (Invitrogen, Carlsbad, Calif., USA) and $0.4 \mathrm{ml}$ of chloroform. This mixture was centrifuged at $12,000 \mathrm{~g}$ for $15 \mathrm{~min}$ at $4^{\circ} \mathrm{C}$, then the aqueous layer was transferred to new tubes. After 1 volume of $700 \mathrm{ml} / \mathrm{l}$, ethanol was added to 1 volume of aqueous layer; the mixture was then applied to a QIAamp MinElute Virus column (Qiagen, Hilden, Germany) and processed according to the recommendations of the manufacturer. Total RNA was eluted 
with $20 \mu \mathrm{l}$ of RNase-free water and directly reverse-transcripted with an Omniscript RT Kit (Qiagen) in accordance with the instructions of the manufacturer. After this, complementary DNA products were amplified by real-time quantitative PCR according to the manufacturer's instructions (QuantiTect Probe PCR Kit; Qiagen) with a 2- $\mu$ l aliquot of complementary DNA and the kit components in a reaction volume of $20 \mu \mathrm{l}$. TaqMan PCR analyses for PLAC1 were performed with predeveloped and commercially available primers and probe sets described previously [8]. As an initial step, we verified that each PCR assay was specific to mRNA and not to genomic DNA. Amplification data were collected and analyzed with an ABI Prism 7900T Sequence Detector (Applied Biosystems). Each sample was analyzed in duplicate, and multiple negative water blanks were included in every analysis. The following thermal profile was used: $15 \mathrm{~min}$ of denaturation at $95^{\circ} \mathrm{C}$, followed by $15 \mathrm{~s}$ of annealing at $94^{\circ} \mathrm{C}$, and $1 \mathrm{~min}$ of extension at $60^{\circ} \mathrm{C}$. Quantification of gene expression was performed by investigators who were blinded to the outcome of pregnancy. The amounts of mRNA samples were expressed in terms of copies per milliliter. To quantify mRNA concentrations, we prepared plasmid DNA for calibration curves as previously described [18].

Statistical analysis was performed at the Department of Medicine and Surgery (DIMEC), Division of Prenatal Medicine at Bologna University. Distributions of demographic characteristics and $\log$ distribution of the multiple of the median (log MoM) PLAC1 mRNA concentrations were analyzed by Student's t test and a $\chi^{2}$ test after testing for normality by with the KolmogorovSmirnov test. The a priori risks [intended as odds/( $1+$ odds $)$, where odds $=\mathrm{e}^{\mathrm{y}}$ ] and $Y$ were calculated using the following equation: $Y=-6.311+0.092 \times \mathrm{BMI}+(0.855$ if woman's mother had $\mathrm{PE}$ or 0 if she did not $)+(-1.481$ if parous without previous $\mathrm{PE}$, 0.933 if parous with previous $\mathrm{PE}$, or 0 if nulliparous). The a priori risks from this formula were subsequently multiplied by a likelihood ratio (LR) based on log MoM MAP obtaining the a priori + MAP risk as reported by Poon et al. [14].

The coefficient relative to the black race was omitted since no black women were present in this series of data.

The receiver operating characteristic (ROC) curve was used to evaluate whether, after log MoM conversion, PLAC1 was associated with a significant risk of PE. The LR associated with the PLAC1 MoM distribution was calculated as reported by Cuckle and Wald [19]. Successively, the a priori + MAP risk was combined with the LR obtained by the PLAC1 distribution in order to calculate a patient-specific risk for PE. A multivariable ROC curve having the patient-specific risk (the output of the logistic regression) as the exploratory variable was constructed and the relative DR was calculated.

\section{Results}

Table 1 reports the demographic characteristics of the series of data stratified according to the generated groups. Table 2 reports the distribution of the variables used to build up the statistical models for PE prediction. As shown in table 2 , the median quoted a priori + MAP risk of $\mathrm{PE}$ is seven times higher for PE cases than controls (5.6
Table 1. Demographic characteristics

\begin{tabular}{lccr}
\hline Variable & $\begin{array}{l}\text { Cases } \\
(\mathrm{n}=43)\end{array}$ & $\begin{array}{l}\text { Controls } \\
(\mathrm{n}=200)\end{array}$ & $\mathrm{p}^{1}$ \\
\hline Maternal age, years & $28.8 \pm 5.46$ & $27.9 \pm 5.34$ & 0.356 \\
BMI & $23.21 \pm 3.74$ & $23.07 \pm 3.50$ & 0.821 \\
Gestational age at & & & \\
$\quad$ enrollment, weeks & $16 \pm 1.46$ & $17 \pm 1.26$ & 0.184 \\
Neonatal weight, g & $2,700 \pm 414$ & $3,190 \pm 488$ & $<0.001$ \\
\% IUGR & 20.9 & 1.5 & $<0.001$ \\
\% LPTD & 16 & 0 & 0.004 \\
Current smoker & 2.3 & 0.5 & 0.229 \\
Woman's mother with PE & 9.3 & 0 & $<0.001$ \\
Previous history of PE & 4.7 & 0 & 0.002 \\
\% nulliparity & 70.5 & 65.1 & 0.623 \\
Weeks at delivery & $37 \pm 1.72$ & $38 \pm 1.35$ & $<0.01$ \\
\hline
\end{tabular}

Data are expressed as the means \pm SD or percentage. ${ }^{1}$ Student's $\mathrm{t}$ test or $\chi^{2}$ test. $^{2}$ Late preterm delivery $(<37$ and $>34$ weeks' gestation).

Table 2. Distribution of the variables included in the study

\begin{tabular}{lccl}
\hline Variable & $\begin{array}{l}\text { Cases } \\
(\mathrm{n}=43)\end{array}$ & $\begin{array}{l}\text { Controls } \\
(\mathrm{n}=200)\end{array}$ & $\mathrm{p}^{1}$ \\
\hline MAP & $86.6 \pm 8.56$ & $80.9 \pm 6.89$ & $<0.001$ \\
PLAC1 log MoM & $0.32 \pm 0.43$ & $0.00 \pm 0.42$ & $<0.001$ \\
a priori + MAP risk, \% & $5.7 \pm 76$ & $0.8 \pm 3$ & $<0.001$ \\
Patient-specific risk, \% & $17.8 \pm 222$ & $1.5 \pm 75$ & $<0.001$ \\
\hline
\end{tabular}

Data are expressed as medians $\pm \mathrm{SD} .{ }^{1}$ Student's t test

vs. $0.8 \%$ ). PLAC1 log MoM was higher in PE cases when compared to controls and associated with a significantly low $\mathrm{p}$ value. A Kolmogorov-Smirnov test yielded $\mathrm{p}>0.05$ for the log MoM distribution in both cases and controls. The distributions of PLAC1 log MoM values in both PE and control pregnancies followed a log-Gaussian pattern at least between -2 and $+2 \mathrm{Z}$-score in the unaffected group, as judged by a probability plot (fig. 1). Table 3 shows that both the a priori + MAP risk and the PLAC1 MoM are associated with a significant discrimination between cases and controls as expressed by the areas under the curve (AUCs). Finally, DRs for all the available models were calculated. The patient-specific-risk model (combination of the a priori + MAP risk model plus MoM PLAC1 model) yielded the highest DR. The best improvement in DR was observed at the FPR of 5\% since it moved from 35.5 to $55.8 \%$. 
Fig. 1. a P-plot of the log PLAC1 MoM values in cases (black, blue in the online version) and controls (gray, red in the online version). On the right side of the frame the correspondent Gaussian distributions are also reported (solid line = cases; dotted ori + MAP risk of late PE obtained by the Poon's model. c ROC curve obtained for log PLAC1 MoM. d ROC curve for the a priori + MAP risk of late PE (lower) and patient-specific risk of late PE (upper) obtained by the combination of the a priori + MAP risk of late PE plus log PLAC1 MoM. line $=$ controls). $\mathbf{b}$ ROC curve for the a pri-
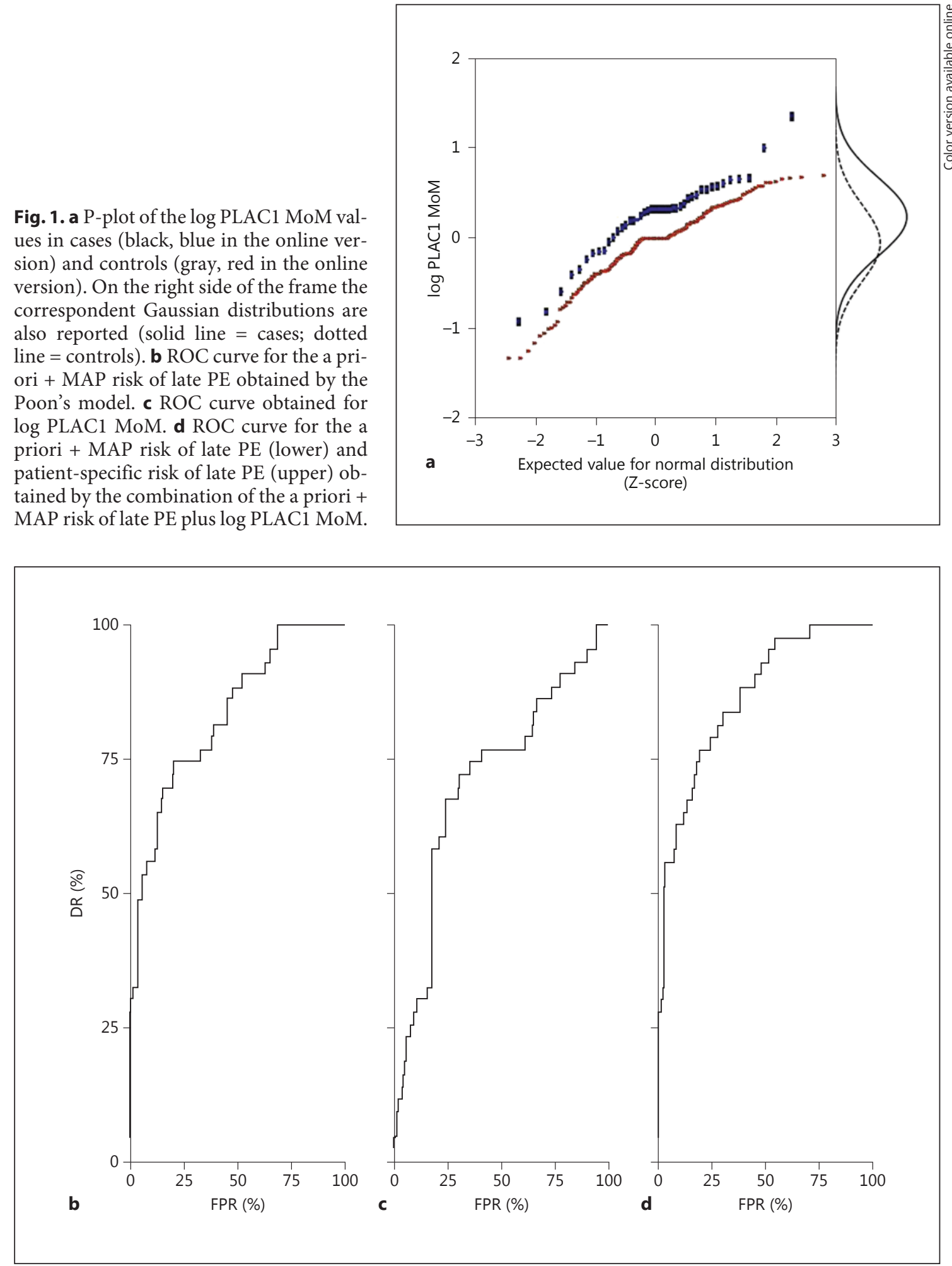
Table 3. Screening for late PE by maternal history + MAP (a priori risk), PLAC1 MoM, and patient-specific risk; comparison of ROC curves and DR at $10 \%$ and $5 \%$ FPR

\begin{tabular}{|c|c|c|c|c|c|c|c|}
\hline \multirow[t]{2}{*}{ Test result variable(s) } & \multirow[t]{2}{*}{ AUC } & \multirow[t]{2}{*}{ SE } & \multirow[t]{2}{*}{$\mathrm{p}$ value } & \multicolumn{2}{|c|}{$95 \%(\mathrm{CI})$} & \multicolumn{2}{|c|}{ DR for fixed FPR } \\
\hline & & & & lower & upper & $10 \% \mathrm{FPR}$ & $5 \% \mathrm{FPR}$ \\
\hline a priori + MAP risk & 0.829 & 0.035 & $<0.001$ & 0.760 & 0.899 & $46.8 \%$ & $35.5 \%$ \\
\hline PLAC1 MoM & 0.706 & 0.047 & $<0.001$ & 0.614 & 0.798 & $30.2 \%$ & $18.6 \%$ \\
\hline a priori + MAP risk + PLAC1 MoM (patient-specific risk) & 0.866 & 0.030 & $<0.001$ & 0.807 & 0.925 & $62.8 \%$ & $55.8 \%$ \\
\hline
\end{tabular}

\section{Discussion}

Given the morbidity associated with PE, an enormous variety of biomolecules have been studied to detect those that show evidence of alteration in the maternal circulation during early pregnancy, before the manifestation of clinical symptoms. Therefore, PE screening is in growing demand, and many papers support the combination of several risk factors to derive a combined method for the patient-specific risk of PE. However, the vast majority of the published results involve biochemical markers and/or Doppler measurements - only a few papers have reported on the predictive values of molecular markers. In previous studies, PLAC1 gene expression has been reported to be higher in PE patients than in controls [13-15]. PLAC1 mRNA concentrations are a function of PE severity and weeks of onset, and are higher in early-onset PE than in late-onset PE [9]. Given this difference in PLAC1 distribution, in this paper we selected only those cases of late $\mathrm{PE}$ that are the greater part of available series of data in order to have a more homogenous sample. The cellular function of PLAC1 is not known, but it is thought to be membrane associated and has been linked to trophoblast differentiation $[6,20]$. It is possible that higher levels of PLAC1 are related to an abnormal interaction between trophoblast and uterine tissues which induces a defective vascular remodeling of maternal spiral arteries leading to noninvasion of trophoblast and placental insufficiency $[21,22]$. Therefore, behind the increase of PLAC1 in PE women's plasma, one possible mechanism could be the enhanced expression of placental mRNA due to genetic deregulation.

This is the first paper that prospectively combines the risk derived from maternal factors like history and MAP, coming from a previously calculated equation generated by a vast number of cases, with a molecular marker of a new data set. Only two studies had previously provided a model based on a panel of mRNA species combined with parity $[12,13]$, but in those only a logistic equation had been retrospectively generated, i.e. by using the same cases recruited in the paper. Many multivariable analyses have been proposed to predict both early and late $\mathrm{PE}$ at the first trimester of pregnancy. In general, there is a trend of using several different logistic regression-based equations for the calculation of the a priori and the a posteriori risk of PE. Some papers have instead reported a mixed model that uses an LR derived from Gaussian distribution of some marker like log Doppler of uterine arteries or log MAP combined with risk logistic-regression or which is derived from a Gaussian model [23].

The model that we used to calculate the a priori + MAP risk yielded a lower DR (at 10\% FPR) than the one reported in the original paper by Poon et al. [14] in their cohort using the same set of predictors (62.5 vs. $46.8 \%$ ), but with quite similar AUC (0.852 vs. 0.829$)$. Similar underestimation has also been recently noted in a study by Scazzocchio et al. [24]. The possible role ethnicity plays in lowering the DR must also be noted. However, irrespective of the performance, the estimated a priori + MAP risk of our PE cases was sevenfold that in controls. Reasons for such an underestimation of the DR are probably due to our population which bears a lower rate of risk factors than that enrolled by Poon et al. [14]. For example, the rate of nulliparity between cases and controls is quite similar in our series of data, while in Poon's paper nulliparity was almost $20 \%$ higher in PE cases [14]. However, the increase in MAP values in our study was similar to Poon's (roughly 6.5 vs. $8 \%$ ).

PLAC1 MoM alone yielded a sufficient DR to be considered in further studies and its addition consistently improved the DR of the patient-specific risk. We dosed PLAC1 in the second trimester since it is definitely detectable at that time [25] and also because the low degree or absence of placental insufficiency typical of late PE makes predicting late $\mathrm{PE}$ in the first trimester quite challenging. Some biochemical markers like PlGF, however, when 
combined with a panel of specific markers, yielded a more promising result in late PE screening, also in the first trimester [23]. To conclude, the present study suggests that screening combining maternal factors with PLAC1 is useful for predicting late PE. Our findings indicate and confirm previous studies on the different levels of mRNA for PLAC1 in PE before clinical onset versus controls. The marker itself has a sufficient DR, but probably cannot be used alone, and a panel of various mRNAs, species, or other strategies like Doppler measurements could be used. Among the limitations of the study, we acknowl- edge that the performance of the screening proposed here should be validated in further prospective studies.

\section{Disclosure Statement}

The authors report no conflict of interest. This study was supported in part by a PRIN project 2007 and Ricerca Fondamentale Orientata (A.F.), and by Grants-in-Aid for Scientific Research from the Ministry of Education, Science, Sports and Culture of Japan (Grant No. 09158522) and from the Ministry of Health, Labor and Welfare of Japan.

\section{References}

1 Duley L: The global impact of pre-eclampsia and eclampsia. Semin Perinatol 2009;33:130137.

2 Lewis G (ed): The Confidential Enquiry into Maternal and Child Health (CEMACH). Saving Mothers' Lives: Reviewing Maternal Deaths to Make Motherhood Safer - 20032005. The Seventh Report on Confidential Enquiries into Maternal Deaths in the United Kingdom. London, CEMACH, 2007.

3 MacKay A, Berg C, Atrash H: Pregnancy related mortality from preeclampsia and eclampsia. Obstet Gynecol 2001;97:533-538.

$4 \mathrm{Ng} \mathrm{EK}$, Tsui NB, Lau TK, et al: mRNA of placental origin is readily detectable in maternal plasma. Proc Natl Acad Sci USA 2003;100: 4748-4753.

5 Cocchia M, Huber R, Pantano S, et al: PLAC1, an Xq26 gene with placenta-specific expression. Genomics 2000;68:305-312.

6 Jackman SM, Kong X, Fant ME: Plac1 (Placenta-specific 1) is essential for normal placental and embryonic development. Mol Reprod Dev 2012;78:564-572.

7 Farina A, Rizzo N, Concu M, et al: Lower maternal PLAC1 mRNA in pregnancies complicated with vaginal bleeding (threatened abortion $<20$ weeks) and a surviving fetus. Clin Chem 2005;51:224-227.

8 Fujito N, Samura O, Miharu N, Tanigawa M, Hyodo M, Kudo Y: Increased plasma mRNAs of placenta-specific 1 (PLAC1) and glial cells missing 1 (GCM1) in mothers with pre-eclampsia. Hiroshima J Med Sci 2006;55:9-15.

9 Purwosunu Y, Sekizawa A, Farina A, et al: Cell-free mRNA concentrations of $\mathrm{CRH}$, PLAC1, and selectin-P are increased in the plasma of pregnant women with preeclampsia. Prenat Diagn 2007;27:772-777.
10 Kodama M, Miyoshi H, Fujito N, Samura O, Kudo Y: Plasma mRNA concentrations of placenta-specific 1 (PLAC1) and pregnancy associated plasma protein A (PAPP-A) are higher in early-onset than late-onset pre-eclampsia. J Obstet Gynaecol Res 2011;37:313318.

11 Akolekar R, Syngelaki A, Sarquis R, Zvanca $\mathrm{M}$, Nicolaides KH: Prediction of early, intermediate and late pre-eclampsia from maternal factors, biophysical and biochemical markers at 11-13 weeks. Prenat Diagn 2011; 31:66-74.

12 Farina A, Zucchini C, Sekizawa A, et al: Performance of messenger RNAs circulating in maternal blood in the prediction of preeclampsia at 10-14 weeks. Am J Obstet Gynecol 2010;203:575.e1-7.

13 Sekizawa A, Purwosunu Y, Farina A, et al: Prediction of pre-eclampsia by an analysis of placenta-derived cellular mRNA in the blood of pregnant women at 15-20 weeks of gestation. BJOG 2010;117:557-564.

14 Poon LC, Kametas NA, Pandeva I, Valencia $\mathrm{C}$, Nicolaides $\mathrm{KH}$ : Mean arterial pressure at $11(+0)$ to $13(+6)$ weeks in the prediction of preeclampsia. Hypertension 2008;51:10271033.

15 Purwosunu Y, Sekizawa A, Okazaki S, et al: Prediction of preeclampsia by analysis of cellfree messenger RNA in maternal plasma. Am J Obstet Gynecol 2009;200:386.e1-7.

16 Brown MA, Lindheimer MD, de Swiet M, Van Assche A, Moutquin JM: The classification and diagnosis of the hypertensive disorders of pregnancy: statement from the International Society for the Study of Hypertension in Pregnancy (ISSHP). Hypertens Pregnancy 2001; 20:9-14.
17 Okazaki S, Sekizawa A, Purwosunu, YIwasaki M, Farina A, Okai T: Measurement of mRNA of trophoblast-specific genes in cellular and plasma components of maternal blood. J Med Genet 2006;43:e47.

18 Purwosunu Y, Sekizawa A, Koide K, et al: Cell-free mRNA concentrations of plasminogen activator inhibitor-1 and tissue-type plasminogen activator are increased in the plasma of pregnant women with preeclampsia. Clin Chem 2007;53:399-404.

19 Cuckle HS, Wald N: Testing using single marker; in Wald N, Leck I (eds): Antenatal and Neonatal Screening. Oxford, Oxford University Press, 1984, pp 3-22.

20 Fant M, Barerra-Saldana H, Dubinsky W, Poindexter B, Bick R: The PLAC1 protein localizes to membranous compartments in the apical region of the syncytiotrophoblast. Mol Reprod Dev 2007;74:922-929.

21 Fisher SJ: The placental problem: linking abnormal cytotrophoblast differentiation to the maternal symptoms of preeclampsia. Reprod Biol Endocrinol 2004;2:53.

22 Luttun A, Carmeliet P: Soluble VEGF receptor Flt1: the elusive preeclampsia factor discovered? J Clin Invest 2003;111:600-602.

23 Akolekar R, Syngelaki A, Poon L, Wright D, Nicolaides KH: Competing risks model in early screening for preeclampsia by biophysical and biochemical markers. Fetal Diagn Ther 2013;33:8-15.

24 Scazzocchio E, Figueras F, Crispi F, et al: Performance of a first-trimester screening of preeclampsia in a routine care low-risk setting. Am J Obstet Gynecol 2013;208:203.e1-10.

25 Heung MM, Jin S, Tsui NB, et al: Placentaderived fetal specific mRNA is more readily detectable in maternal plasma than in whole blood. PLoS One 2009;4:e5858.
Circulating mRNA for PLAC1 in the Screening for Late Preeclampsia 